\title{
Biomimetic Designs for Automobile Engineering: A Review
}

\author{
I.D. Wijegunawardana ${ }^{1,2, *}$ and W.R. de Mel ${ }^{1}$ \\ ${ }^{1}$ Faculty of Technology, University of Sri Jayewardenepura, Pitipana, 10200 Homagama, Sri Lanka \\ 2Department of Mechanical Engineering, University of Moratuwa, Katubadda, 10400 Moratuwa, Sri Lanka
}

\begin{abstract}
Automobile engineering often demands creative and innovative concepts to achieve their performance and efficiency targets. However, the strategies used to create these concepts are evolving with time. Recently, heightened attention has been noticed to using biomimetic concepts in the field of automobile design. The studies that apply biomimicry to automobile design often exhibit improvements in vehicle performance and fuel efficiency. Consequently, biomimetic concepts have extended an opportunity to the automobile industry to generate futuristic designs with advanced technology. However, the innovations and inventions in biology are usually published with more complex terminology that is not convenient for the research community working on automobile engineering. Hence, a notable delay is present in transferring the recent discoveries in biology to engineering researches so that they can develop strategies to mimic them. The purpose of this paper is to provide a brief overview of the biomimetic design approaches that have been and can be used in automobile design. In addition, the paper includes a classification of biomimetic concepts, which reveals the current research interests and highlights the areas with a deficit of research that is required to be addressed extensively in the future.
\end{abstract}

\author{
ARTICLE HISTORY \\ Received: 26th Jan 2021 \\ Revised: 25 ${ }^{\text {th }}$ Aug 2021 \\ Accepted: $6^{\text {th }}$ Sept 2021
}

\section{KEYWORDS}

Biomimicry;

Nature-inspired;

Design;

Automotive;

Automobile

\section{INTRODUCTION}

Biomimetics, in other words imitating life, is a creative form of technology that mimics nature in various developments that improve the quality of life of people. Biomimetics is not a novel concept but has been used since the beginning of human inventions. In recent times, extensive engagement of biomimetics has been noticed in the development of products and behaviours in the fields of science, commerce, and arts [1]. At the moment, the research community seek to develop systematic methodologies to practice biomimetic concepts in various disciplines. For instance, Nagel et al. [2] introduce a methodology to teach the designers to implement the bio-inspired design in engineering developments using the Concept of Knowledge $(\mathrm{C}-\mathrm{K})$ theory. This method demonstrates the capability of improving the creativity and imaginative power of engineering students. On the other hand, biological organisms have been surviving on this earth, evolving and adapting for 3.8 billion years to their habitats [3]. As a result, biological species possess well-optimised structures and behaviours to achieve different tasks in their environment. Hence, the study of nature provides the best design options when designing a machine that carry-out the tasks which are currently done by biological organisms.

Since the beginning of the automobile industry, designers have adopted the shapes of birds and fish when developing optimised shapes to overcome aerodynamic resistance. Nevertheless, recently, an abundance of automobile components such as wheels, cooling fans and lubricants were identified in the literature developed using the biomimetic design approaches. Here, biomimetic designs have reduced their limitations in performance and fuel economy while enhancing additional features such as appearance and passenger ergonomics [4], [5]. Biomimicry concepts can be used in different stages of automobile development, such as design, manufacturing, and control. Predominantly, these concepts are based on macro structures, microstructures, and behaviours of biological organisms. Here, macro/micro-structures are mimicked in mechanical designs while behaviours are used in the development of control systems and optimisation processes [2].

The modern automobile developments with intelligent systems often demand advanced control algorithms. To meet these demands, the natural behaviours of different animals have been adopted for optimisations and in decision making. Consequently, particle swarm optimisation (PSO), ant colony optimisation (ACO), Artificial Immune Systems (AIS), African Buffalo Optimisation (ABO), and dragonfly optimiser have come to the forefront as bio-inspired optimisation algorithms. These were, for the most part, used in optimising automobile safety requirements [6], traffic control [7], vehicle routing [8], manufacturing processes [9], and various design optimisations [10]. Moreover, in some instances, intelligent automobile systems also have mimicked the vision capabilities that the biological organisms possess [11].

A major concern noticed in using biomimetic concepts in the field of automobile engineering is the shortage of mutual research platforms that links biological innovations/discoveries with automobile engineering. Although there are numerous publications on discoveries in biology, they are frequently isolated for engineers. Two major reasons for that are the imaginative nature of biology; and the uncommonly used (Latin) taxonomy. Yet, in recent times, few publications have been identified that discussed some specific applications of biomimicry in automobile-related areas. These include reviews on tribology [12], topology optimisation of mechanical designs [13], and aerodynamics [4]. 
This paper briefs the state of the art research and developments that incorporate biomimicry into automobile design. However, this review has limited the discussion to biomimicry in the development of ground vehicles used in transportation tasks. Thus, automotive systems such as construction vehicles, trains, ships, and air vehicles will not be discussed in this article. Automobile-related developments inspired by biological behaviours are also excluded from the review. This will only discuss the biological inspiration for mechanical designs. Nevertheless, the authors expect that this review will be useful for automobile researchers to develop rather efficient and high-quality vehicles in the future. This paper is structured as follows. Initially, the paper introduces a classification of biomimetic research, highlighting the scope of this review. Then, the biomimetic macrostructures used in vehicle designs have been explained. Subsequently, different automobile features based on biomimetic microstructures have been explained. Then, the design approaches used in biomimicry has been discussed, highlighting the future directions of this field. The final section of the article provides a brief conclusion of the review.

\section{CLASSIFICATION OF BIOMIMETIC DESIGNS IN AUTOMOBILES}

Applications of biomimicry have been adopted by different fields. Therefore, the methodology proposed in Figure 1(a) was used when filtering the research that falls within the scope of this analysis. Initially, through the classification, automotive-related research based on biomimicry has been selected. In the next step, biomimicry research was further narrowed down to the automobile-related applications for regular transport vehicles.

(a)

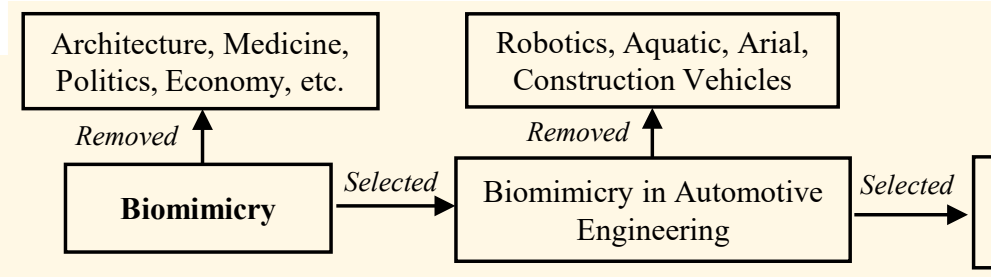

(b)

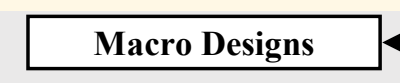

\section{Automobile Body}

- Vehicle body overall shape (boxfish [16], tiger beetle [17], avocado-pear [18])

- Dimpled surface (shark skin [20-24])

- Flow control devices (secondary feathers of a bird's wing [30], tubercles effect of humpback whales [27], fore-body of a sea lion [26])

- Safety attachments (diatom [33], honeycomb [34])

- Lightweight structures (beetle elytra[29], honeycomb [31], marsh horsetail, tortoise shell, spider web [32])

\section{Wheel Assembly Components}

- Helical shaped spring (tendril structure [40])

- Hydraulic damper (abalone shells[41])

- Non pneumatic tires (honeycomb [36,37])

- Tires profiles (cheetah paw [38], torrent frog[38, 39])

- Break disc (wings of the owl \& surface of the locust [44])

\section{Powertrain \& Under-the-hood Components}

- Cooling system for EVs (human respiratory system[49])

- Shaft seal for EV coolant pump ( natural lubrication in Synovial joint [48])

- Cooling Fans (ribbed ridges in animals [47])

- Adsorption Bed for AC system (honeycomb [50])

- Diesel particulate filters (honeycomb [51]) (c)

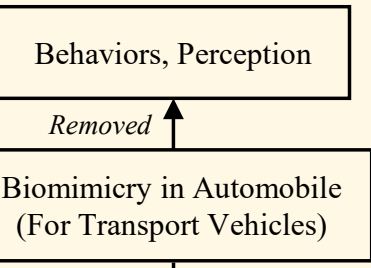

Selected

Biomimetic Structures

\section{Surface Coatings}

- Drag reduction (lotus leaf, shark skin [54,84])

- Self cleaning (lotus leaf[55], Photocatalysis[56], tear film of the human eye[57])

- Self healing (Human skin[67,68])

- Wetness resistance (surface of a biological organism $[55])$

- Scratch resistance (Mollusc shell[55])

- Color changing (Chameleon[83])

- Transparency (Human Eye [57])

- Corrosion resistance (petal of rad rose [61])

- Lubrication (Pitcher plant, Salvinia[62])

\section{Other Methods}

- Wear resistance (Bionic Surface[69-72])

- Lubrication (Synovial fluid in human joints [73,74])

Figure 1. Classification of biomimetic designs used in automobile developments: (a) Article scope, (b) biomimetic macro-structures, (c) biomimetic micro-structures. 
Finally, the research based on the micro or macro mechanical structures was selected, excluding the research based on behaviours of the biological organisms. Here, macro-level biomimetic designs have been categorised further into three clusters. The basis of this classification was the sub-system of the automobile (body, wheel assembly, powertrain \& under-the-hood), which embraced the biomimetic design, see Figure 1(b). Microstructural mimicry, on the other hand, provides materials with enhanced properties. During the analysis, these materials were identified in several automobilerelated applications. They were adding futuristic features to the automobiles such as self-cleaning, self-healing, scratch resistance, drag reduction, and lubrication, see Figure 1(c). A summary of the identified literature on biomimetic macro and microstructures is illustrated in Figure 1(b) and 1(c).

\section{REVIEW OF BIOMIMETIC STRUCTURES}

The literature resources were extracted from the research databases such as Scopus and IEEE Xplore. Moreover, the web resources were explored to extract the details of the related products in the commercial stages. The selected research are presented in this section adhering to the two classes: macro-scale design solutions and micro-scale design solutions.

\section{Biomimetic Macro Structures in Automobile Designs}

The macro-structural design solutions in this section include the biomimetic implications on the overall structure of the vehicle. It also discusses biomimetic components in automobile sub-systems such as wheel assembly, powertrain, steering, and air conditioning.

\section{Biomimetic developments for automobile body}

Since the mid of $19^{\text {th }}$ century, several automobile producers have used animal appearances for their models. They have also used the mimicked animal name as the model name. These include the Ford Mustang, AMC Marlin, Shelby Cobra, Plymouth Barracuda, Chevrolet Cheetah, and Volkswagen Beetle [14] (see Figure 2). Initially, the main expectation of using nature-inspired shapes was to develop low aerodynamic drag in automobiles. Consequently, this approach has also enhanced customer attraction. For example, Hou et al. [15] explain how animal-inspired shapes and their names can physiologically influence automobile users.

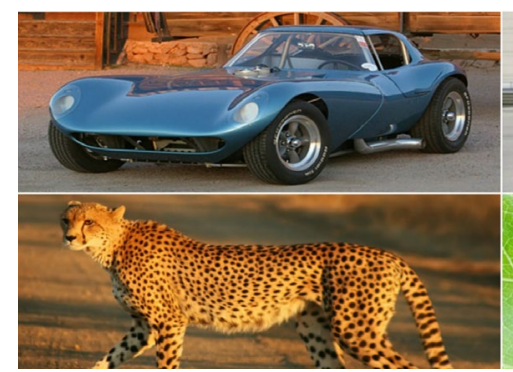

(a)

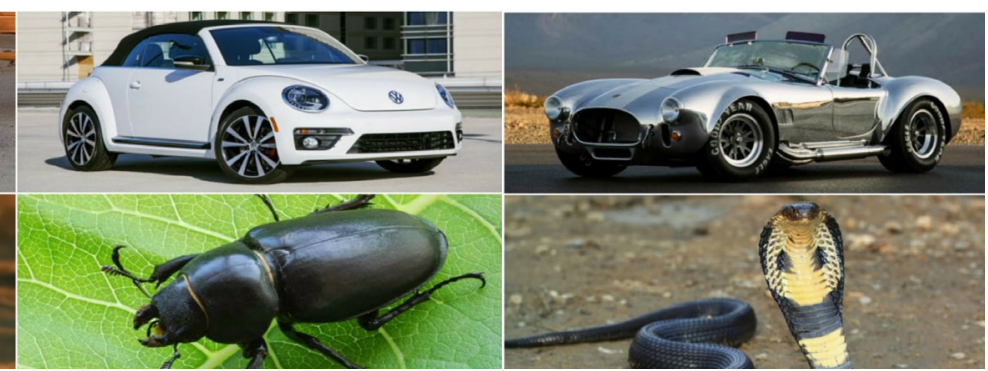

(b) (c)

Figure 2. Animal inspired shapes for automobiles[14] (a) Chevrolet Cheetah, (b) Volkswagen Beetle, (c) Shelby Cobra.

More recently, the Mercedes-Benz company has introduced a vehicle named bionics car [16], which had a shape that was inspired by a boxfish, see Figure 3(a) to 3(c). This shape has proven to have an extremely low drag coefficient. In another attempt, Peng et al. [17] managed to design a low-drag automobile using the shape of a tiger beetle, see Figure 3(d) and 3(i). With this imitation, the design has specifically reduced the after-body drag. Another example of this is the avocado-pear-inspired vehicle design introduced by Agyei et al. [18]. These developments imply that the bionics concept is expected as the foundation of future automobile design [19].

On the other hand, the drag resistance on the vehicle can be reduced by changing the texture of the external surface of the automobile body. In the classical engineering mindset, it is believed that smooth surfaces are associated with streamlined flow and low drag. However, in nature, the creatures that fly or swim are, for the most part covered with feathers or scales and possess a surface texture that varies in frequency and amplitude. For instance, the scales present in the microstructure of the shark's skin is depicted in Figure 3(j). This surface texture can dramatically affect aerodynamic drag and manoeuvrability [5]. This concept was further proved by the dimpled surface texture adapted by the golf ball in the early 20th century. Similarly, automobile designers also started including biomimetic textures in their body designs to improve vehicle aerodynamics [20]. Lexus [21], for example, has introduced dimples on their luxury sedan, claiming a reduction in noise and drag.

Moreover, a vinyl vehicle-wrap system applied on all the painted surfaces named MPG-Plus has been introduced by Fastskinz Inc, see Figure 3(1). This has closely spaced shallow dimples with about a $5 \mathrm{~mm}$ diameter [22]. The company claims this improves aerodynamics and fuel efficiency. Another similar design was adapted in the solar panel integrated roof of the concept car shown in Figure 3(m), which was introduced by the French designer, Dimitri Bez [23]. A dimpled surface was also investigated by Kashyap et al. [24], where the spherical depressions were introduced to the bonnet of a hatchback car. The article has analysed the design with CFD and reported a significant reduction in the drag coefficient. When considering the concept of dimpled surface, it is clear that the spacing and amplitude of the bumps need critical 
attention as same as the range of flow velocities across the surface. If not, it is more likely to increase drag and noise rather than contribute to their reduction.

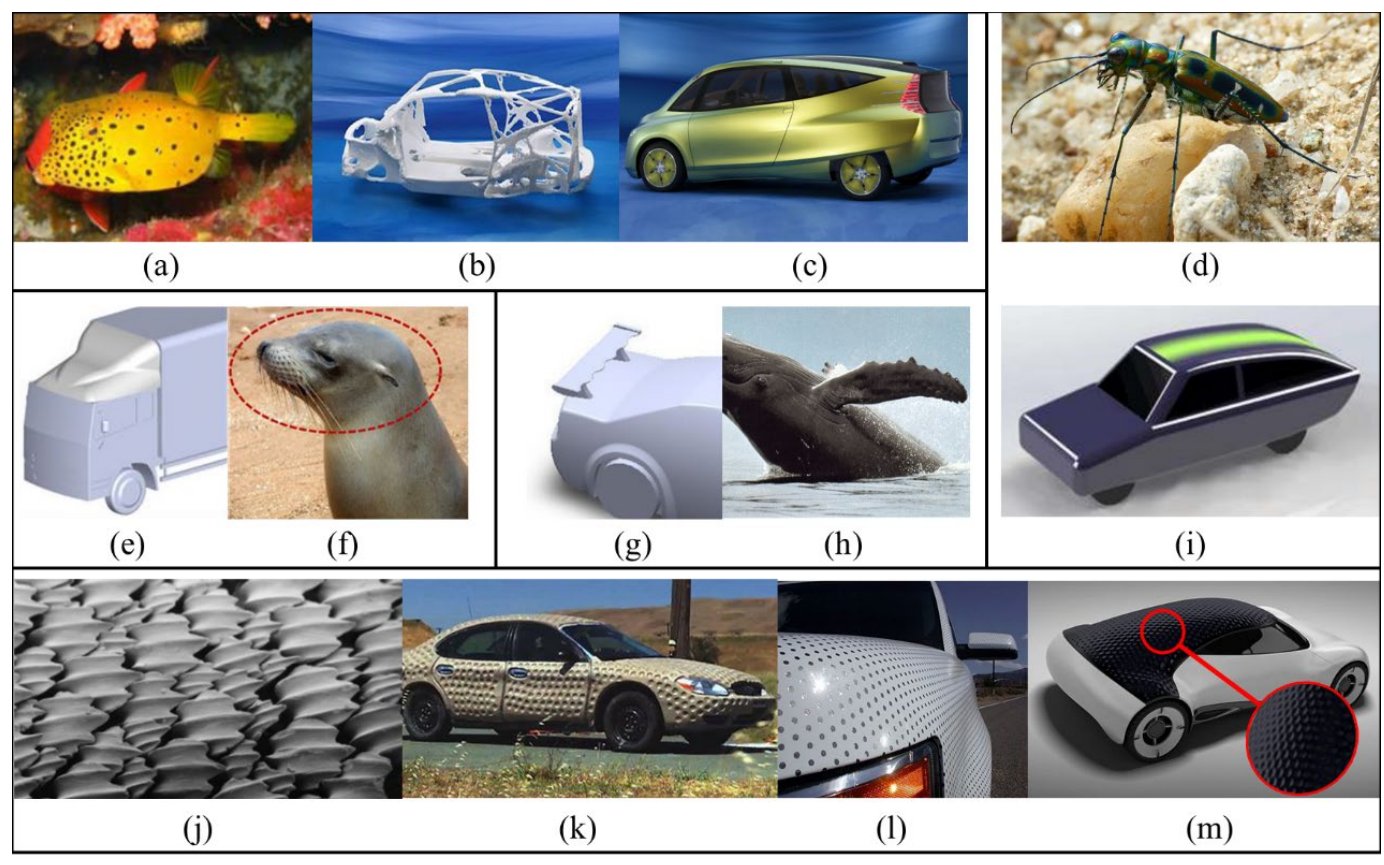

Figure 3. Biomimetic macro-scale designs for aerodynamics improvement: (a) boxfish [1], (b) skeleton structure of blowfish [1], (c) Benz bionic car [1], (d) tiger beetle [25], (e) cab-roof fairing [26], (f) sea lion fore-body[26], (g) rear spoiler [27], (h) tubercles effect in whale [28], (i) tiger beetle inspired design [17], (j) magnified shark-skin [84], (k) dimpled vehicle surface [20], (1) vehicle-wrap [22], (m) Hexa car [23].

Other than the basic structure, automobiles contain additional attachments that perform flow controlling to improve aerodynamics. According to the literature, these items have been optimised with the influence of biomimicry. For example, Kim et al. [30] explain how the device called automatic moving deflector (AMD) inspired by the secondary feathers of a wing of a bird has contributed to the drag reduction. According to this research, AMD has delayed the separation point by keeping the flow attached to the moving deflector. Thus the device was able to obtain a maximum of $19 \%$ drag reduction. Ghaffar et al. [27] proposed a novel design inspired by the tubercles effect for the rear spoiler, see Figure 3(g). This effect has been mimicked from the pectoral flippers of humpback whales, as shown in Figure 3(h), which provides them exceptional acrobatic manoeuvrability [28]. The modified spoiler has shown a reduction of drag and improved aerodynamic performance through virtual simulations. Heavy-duty transport vehicles such as trucks are known to be severely affected by aerodynamic drag. Therefore, a multitude of additional components has been developed on behalf of those. According to the literature, it was identified that recently these components had been designed based on the shape of biological organisms. Kim et al. [26], for instance, have introduced a cab-roof fairing inspired by the external fore-body shape of a sea lion, see Figure 3(e) and 3(f). This research further claimed that the fairing had reduced the aerodynamic drag by at least $20 \%$.

Other than the drag reduction, vehicle designers have also adapted some structures from the biological organisms to improve the strength of the vehicle body. For example, the structural changes in the body panels were identified to be obtaining improved strength. The most popular technique for this is honeycomb-based structures. Fan et al. [31] is one of the few research that introduced a composite material with a honeycomb based structure. This is known as the honeycomb sandwich structure and is produced by sandwiching two high-strength skin panels with one layer of honeycomb core through adhesives. The research claimed that this material has lightweight, high specific strength, high specific stiffness, vibration-resistance, and heat-insulation. It further claimed to be applicable for automobile components such as body panels, frame structures, bumpers, seats, and doors. In another research, Guo et al. [29] explain how to design three bioinspired lightweight structures based on the cross-section of a beetle's elytra. It further claims that a bio-structure with spherical holes and hollow columns perpendicular to the top and bottom surfaces best imitates the microstructure of the Cybister Elytra and has the greatest strength against compression and bending. Wang et al. [32] also analysed the crashworthiness of several biomimetic patterns of hexagonal prismatic tubes. These were numerically investigated and found that they are superior to the existing designs. In this analysis, bee honeycomb, marsh horsetail, tortoise-shell, and spider web were taken as the references for the bio-inspired structures. In the evaluation, out-of-these, the spider web structure was identified as the strongest.

The body attachments with the purpose of protection during the crash, such as bumpers and crash pads that were developed based on the biomimetic designs, have been occasionally reported in the literature. With the incorporation of biomimetic features, they were proved to have improved crashworthiness. For instance, Hundertmark et al. [33] introduce a crash pad inspired by diatom, which absorbs energy in the case of lateral collisions. The three undulations of the proposed crash pad are of different heights and provide for a sequential absorption of the impact energy. Thus, the 
structure prevents catastrophic damage in a crash. On the other hand, Ganilova et al. [34] provide information about a honeycomb inspired energy-absorbing structure for an automobile bumper. After a mild collision, this structure recovers its shape back and thus become a re-usable solution.

\section{Bio-inspired wheel assembly components}

The wheel assembly components are involved in handling the tire load and tire orientation in an automobile. In order to handle larger loads, these components are required to be made strong. This selection of strong materials consequently contributes to the net weight of the vehicle with a significant amount. The wheel assembly in this article considers the wheels, brake components, suspension components, hubs, and uprights.

According to the literature, non-pneumatic tires have been an extremely popular futuristic concept that has improved the performance of the vehicles while reducing their weight [35]. For these tires, the honeycomb structure has been used on most occasions since it can stand for large stresses [36], [37], see Figure 4(k). These tires also have high deformation capability so that the ride comfort of the vehicle can be increased extensively. Currently, these types of non-pneumatic tires are used in off-road vehicles as well. There have been several research attempts to improve the tire profile based on the biomimetic structures; so that the tire grip can be increased and the rolling resistance can be decreased. Ivanović et al. [38] have introduced two such tire productions. One of them has adopted the biology of the cheetah paw as shown in Figure 4(d), to improve the tire friction, and the other one mimicked the toe pad of the tree frog, see Figure 4(e), to improve the performance on wet surfaces. During running, the paws of the cheetah are narrow and produce low friction, see Figure 4(f). Therefore, it facilitates low energy consumption. During slowing down and changing the running direction, their paws broaden. This technique increases its friction and stability. Hence, the cheetah's paws are optimised for acceleration, efficient changing of the direction, and high stability in curves. The dry tire mentioned in [38] uses this technique possessed by the cheetah. It has the same width as the conventional tire, but it widens during braking, reducing the stopping distance by up to $10 \%$. The other tire explained in the [38] is capable of rolling on wet surfaces without slipping. This wet tire was designed to mimic the toe-pad-microstructure of a tree frog. Barnes et al. [39] has also explained the ability of tree frogs to remain adhered to the wet surfaces. The microstructure of the tree frog toe consists of hexagonal shaped cells separated with thin grooves, see Figure $4(\mathrm{~g})$. When the frog is on a wet surface, these grooves allow fluid to pass through, creating a thin fluid layer in between the pad and surface that sticks the pads to the surface by wet adhesion.

On the other hand, some innovations in the suspension components based on biomimicry were identified in the literature. For example, Kaluvan et al. [40] introduce a spring-damper system that has a tendril structure inspired by the bio climber plant, see Figure 4(a). The key enabling concept of this work was to design a smart spring damper using magneto-rheological (MR) fluid that has variable damping capabilities. The spring is made of a hollow tube as shown in Figure 4(b), which contains the MR fluid, and the tube is winded with a coil, see Figure 4(c). To change the damping constant and the stiffness, the properties of the MR fluid can be altered by changing the properties of the electricity flows through the winding. Yang et al. [41], have proposed a biomimetic damper with lots of potential for futuristic designs. This damper is inspired by the biological bonds present in the abalone shells. This biological technique adapts the biomechanism of sacrificial bonds and hidden length to dissipate energy. Sacrificial bonds are additional, weak but reformable bonds that break before damaging the strong bonds that hold the structure together. When each sacrificial bond brake, the main strong bond will get the freedom to extend further; this is known as the hidden length [42]. When several molecules participate together in a distributed passive damping, the corresponding force-displacement curves become smoother [41]. Here, the resulting forcing behaviour can be readily implemented in dampers for structural control. Therefore, this mechanism claims more efficient than the dashpots and springs used in conventional dampers. The biomimetic damper utilises hydraulic components that allow large damping forces. In order to achieve the initial damping behaviour inspired by the sacrificial bonds, a small volume of air can be added to the system. The air bubble in the hydraulic fluid prevents hydraulic shock when the displacement is both small and closer to the equilibrium position. The damping occurs due to the air compliance generated by a gradual increase in loading. Generally, in animal bones, there will be no negative displacements. Yet, the design of this damper has accounted for both positive and negative displacements [41].

In [44] a brake disc has been designed according to the wings of the owl and the surface of the locust, which reduced the noise and improved the wear resistance of the brake disc [See Figure 4(h)]. In order to achieve this optimised design, the article proposes a modelling platform that can map multi biological functions and produce a conceptual design that follows both references. When designing a vehicle with good performance and fuel economy, weight reduction is a major concern. Here, the weights of wheel assembly components are considerably high since these components are made with heavy metal to deal with larger loads. Therefore, obtaining strong and lighter structures is a critical requirement in an automobile. To achieve this, topology optimisation is commonly used in automobile design with biomimetic reference models. In literature, several instances have been identified [13], [45] where the biomimetic topology optimisations were driven for the suspension arms, brake paddles, brake callipers, and uprights, see Figure 4(i) and 4(j). 


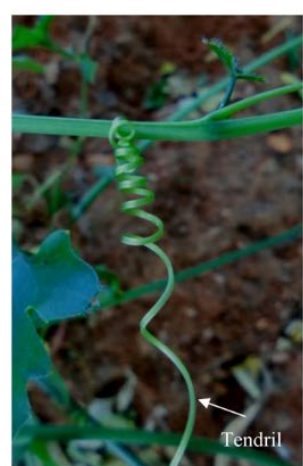

(a)

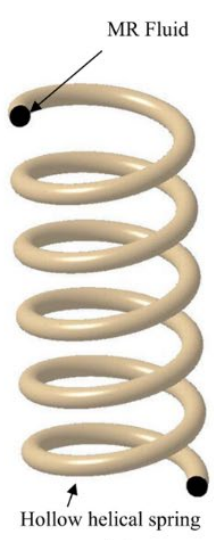

(b)

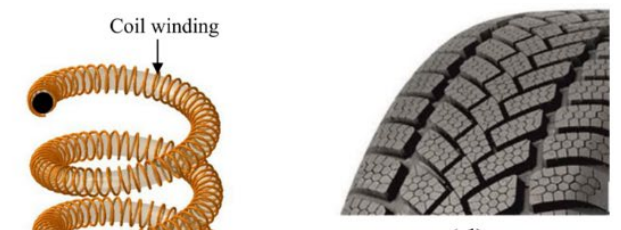

(d)

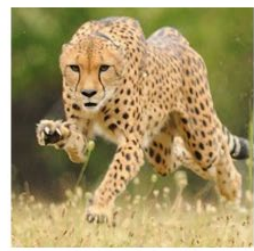

(f)

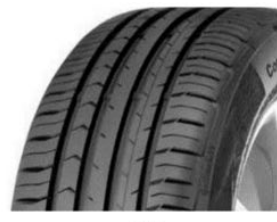

(e)

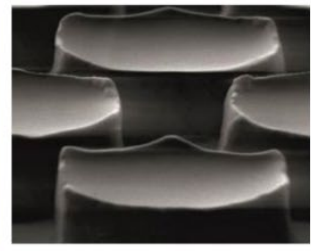

(g)

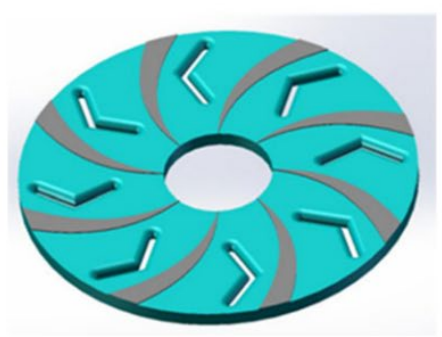

(h)

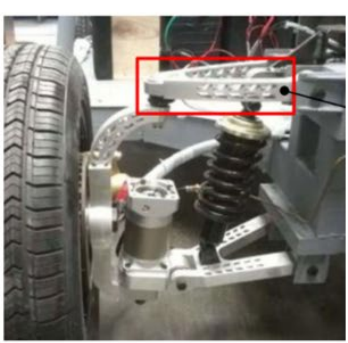

(i)

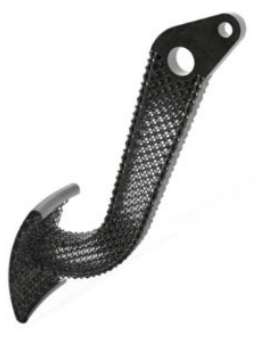

(j)

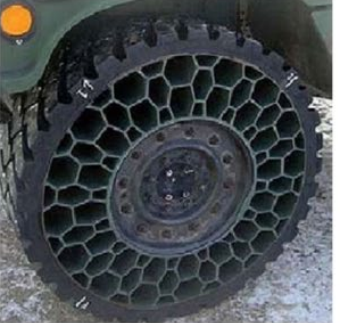

(k)

Figure 4. Automobile wheel assembly related designs inspired by nature: (a), (b), (c) biomimetic spring-damper system [40], (d) dry tire [38], (e) wet tire [38], (f) narrowed paws of a running cheetah [43], (g) magnified tree frog feet [38], (h) bio-inspired brake disk [44], (i) topology optimised suspension arm [45], (j) lattice optimised break paddle arm [13], (k) honeycomb inspired tire [35].

\section{Bio-inspired powertrain and under-the-hood components}

The powertrain can be considered the heart of an automobile. Most of the parameters that become obstacles to higher fuel efficiency and better performance are present in the powertrain. When it comes to nature-inspired concepts, it is the hardest system to be mimicked from biological organisms. However, with the principle of biological energy conversion, recently, hydrogen-powered solar vehicles, with water and sunlight as fuels, has become a growing research field [46].

Although biomimetic in power generation is rare, the research on applying biomimetic design tools to important components and sub-systems in automobiles can be occasionally found in the literature. These include components such as cooling fans, rotary impellers, heat exchangers, pumps, nozzles, and hulls. Here, the cooling system, which plays a major role in sustainable power generation, has got more attention in terms of utilising nature-inspired designs. For example, Wang et al. [47] have introduced a new design for a cooling fan that was able to reduce the noise. Furthermore, in [48], there is a seal lip for an electric vehicle cooling pump that was developed based on a biomimetic design. The seal lip plays a major role in separating the water base coolant from the air in the environment. Here, the hydrated lubrication mechanism found in natural articular cartilage has been adopted in the design of the seal lip. The article claims that the bioinspired seal lip has successfully isolated the coolant from the air and excreted low friction on the shaft compared to the conventional oil seal. Another research performed by Ki et al. [49] has proposed a liquid cooling module that was inspired by a human respiratory system. This was developed to provide enhanced thermos-hydraulic performance. The human respiratory system performs blood circulation while removing carbon dioxide and adding oxygen. The system proposed by $\mathrm{Ki}$ et al. [49] acts the same way, circulating the coolant and remove heat through absorption. Coolant circulates through a plate made from copper foam, mimicking the alveoli structure in the human respiratory system to absorb the heat.

On the other hand, literature has records of biomimetic designs of heat-powered adsorption cooling systems used in automobile air conditioning. This cooling technology is generally beneficial in automobile air conditioning when waste heat is available. Here, in the heat absorption, using honeycomb structure for designs of adsorption beds has shown admirable merits of low cost, low weight, and good heat transfer properties [50]. The honeycomb structure is also identified in the literature for the diesel particulate filters [51]. These systems were used to fulfil emission regulations on soot.

\section{Application of Materials with Biomimetic Micro Structures}

Apart from mimicking the biological structures for the macro designs, the literature also provides details on the usage of microstructures of the biological organisms to develop the mechanical properties of substances. In this section, the microstructures of biological organisms that inspired automobile-related designs are discussed. With the improvement of micro/nano-technology, there have been attempts to modify material structures by chemical deposition and material 
removal methods to obtain biomimetic features [52]. With the help of these engineered material structures, bio-inspired coatings for automobile-related applications has been introduced. These coatings can enhance important qualities in tribology such as wear resistance, wetness resistance, self-healing, self-cleaning, and drag reduction [3]. Therefore, by applying these coatings on the interior or exterior of the surface in the components of automobiles, more futuristic attributes can be obtained.

\section{Drag reduction}

The microstructure of the shark's skin is one of the major contributors to drag-reducing surfaces. The scales present in the shark's skin; see Figure 3(j), instead of a smooth surface, give the ability to keep the fluid flow attached to the body by preventing the generation of vortices that can create a drag [53]. Even though this effect has been used in macro-scale as dimpled surfaces for automobiles, this was merely found in research as a microstructural implication. In literature, a research publication was identified where Baier et al. [84] had introduced an artificial 3D printed microstructure inspired by shark skin for the exterior surface of a race car. The proposed microstructure has the ability to keep the flow attached to the body surface, and thus reduce the drag. This article has further mentioned that the CFD simulations on the modified surface have resulted in a $15 \%$ drag reduction. A commercial manufacturer, CHOOSE NanoTech [54], has developed a drag reduction coating for automobile-body inspired by shark's skin. This product is currently under experiment.

\section{Self-cleaning}

The most popular microstructural wonder, the lotus effect, is performed by the lotus leaves when water effortlessly rolls off the leaf while keeping it clean, without making it wet. This effect is an attribute of the superhydrophobic surfaces. The lotus leaf has obtained this property as a result of the microstructure of its surface. On its surface, instead of a smooth geometry, there are hump-like micro-scaled structures (about 15-20 $\mu \mathrm{m}$ high and 20-25 $\mu \mathrm{m}$ apart); see Figure 5(f), which traps the air and prevent water from spreading on the surface [55]. The other feature that contributes to the superhydrophobic behaviour is the wax present on the surface which enhances hydrophobicity. Due to these features, when water drops onto the hydrophobic surface, it allows the water droplets to form beads, see Figure 5(e), and when the surface is tilted, roll away under gravity while keeping the surface dry. Moreover, as the water droplet rolls off, the dirt particles on the surface of the lotus leaf stick to the water beads and make the surface clean [56]. This process has been illustrated in Figure 6(b). The hydrophobicity of the surfaces can be evaluated through the contact angle of the water droplet on the surface. Figure 5(a) to 5(d) depict four main phases of hydrophobicity of the surfaces (superhydrophilic, hydrophilic, hydrophobic, and superhydrophobic) along with the respective range of the contact angle for each phase.

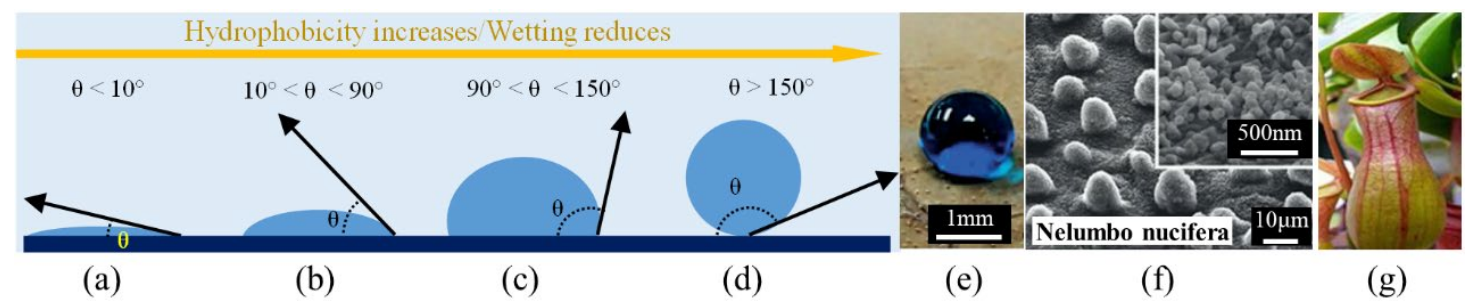

Figure 5. (a), (b) Water contact angles for deferent surface structures, (e) lotus effect [53], (f) micro and nano structures of the lotus leaf [53], $(\mathrm{g})$ pitcher plant [57].

Nevertheless, nature has shown the self-cleaning property even with the hydrophilic surfaces. This was achieved by the super-hydrophilic surfaces in plants such as pitcher plants; see Figure 5(g), and through photocatalytic oxidation property. In these instances, they possess slippery surfaces to remove contaminants or chemical compounds that have the ability to digest organic compounds with the help of UV light [56]; see Figure 6(a). There are several projects to be found in literature where these features have been mimicked in the automobile components such as windshields [57]. Another source that became an inspiration in engineering design is the tear film in the human eye. The tear film has the capability to wash away the dirt and also has a transparent property. Currently, $\mathrm{TiO}_{2}$ is identified as a material that can be used in engineering design to mimic the features of the human tear film [57]. During the analysis, few commercial products were found that incorporate the self-cleaning property into the automobiles. For example, the Nissan automobile manufacturer

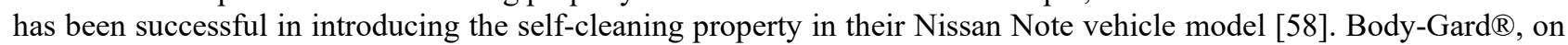
the other hand, is a commercially available protection coating for automobile interior/exterior inspired by the microstructure of the sharkskin [59]. 


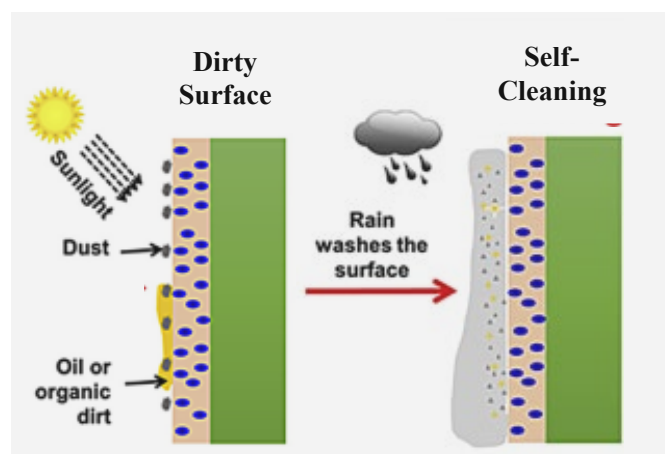

(a)
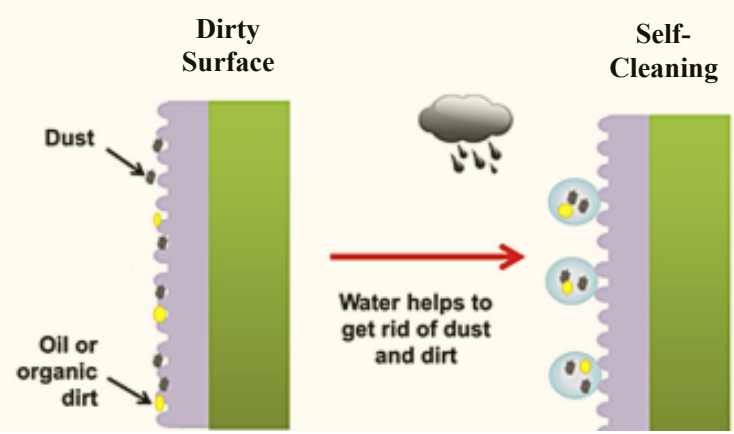

(b)

Figure 6. Self-cleaning coatings [60]: (a) self-cleaning effect of the photo digestion of the super-hydrophilic surfaces, (b) self-cleaning effect of the superhydrophobic effect.

Literature has also provided evidence on research conducted for anti-corrosion based on the superhydrophobic property. For example, Liu et al. [61] explain the two-step methodology (laser and etching) to improve the anti-corrosion feature in a hydrophobic surface that is fabricated on magnesium alloy. The micro-nano structure of the surface proposed in this article, with microscale crater-like and nanoscale flower-like arrangements, is inspired by the petal of a red rose. The article claims that the superhydrophobic surface has improved the corrosion resistance (by reducing wettability) of the magnesium alloy which is commonly used for automobile bodies. In addition, Xiang et al. [62] have reported a development of a slippery surface inspired by the pitcher plant that has the anti-corrosion feature where a lubricant layer protects the metal surface.

\section{Self-healing}

The scratch resistance is also an extremely important feature that is being researched for interior as well as exterior coatings in automobile development. This can keep the appearance of the vehicle for a longer period, reducing the frequency of repairing and thus reducing the maintenance cost. The resistance for the scratches can be developed with different methods [63]-[65]. From them, integrating the self-healing capabilities has been identified as a trending biomimetic solution. Self-healing is an important property existing in nature that has been investigated for application in engineering designs [66]. The self-healing present in the biological organism functions with the involvement of blood and capillaries. When there is an opening (cut) in the human skin, the capillaries that are damaged by the cut will fill the opening with blood and then solidify due to the components present in the blood. Then the skin tissue will be healed gradually with time. A similar process has been mimicked by the recent artificial self-healing materials. These materials contain microcapsules, or hollow fibres that contains a healing agent. Once they have been subjected to a cut or an opening, the agents released and filled the opening in the material.

Recently a few concept cars developed with futuristic designs (i.e. Lamborghini Terzo Millennio [67]) have also been identified that mimicked the self-healing property [60] that biological organisms possess. In addition, the Nissan motor company has developed a biomimetic coating named Scratch Guard which can be applied to the automobile body that provides the self-healing capability [68]. This coating has the ability to recover from the damages without any external intervention, as same as the natural organisms. The methodology used to obtain this capability is using a special highly elastic resin in the basic structure that has high flexibility. This has also increased the strength of the vehicle body due to the increment of its density.

\section{Wear resistance}

Although generally, for automobile components, biomimicry has been hosted with introducing additional coatings to the surfaces, modified properties also can be obtained by altering the original surface structure of components with micro material removal processes. One good example of this is the laser treatment of the surfaces made from cast iron when fabricating brake drums [69] and brake pads [70], to improve the wear resistance capabilities. This method brings out the bionic surface with different shapes, structures, and soft and hard tissues similar to the surface of a biological organism [71], [72].

\section{Lubrication}

For the most part, lubricants that are currently being used for automobile tribology are oil-based and rely on chemical additives. The primary uses of lubricants are wear reduction, friction optimisation, heat removal, carrying away impurities, and wear debris [12]. Although these requirements are fulfilled by the boundary lubrication with oils, the reactions that occur as a result can cause negative effects. While this issue requires the lubricants to be changed frequently, the absence of recyclability of the automobile lubricants causes environmental issues. Therefore, for these reasons, the researchers are now motivated to look toward nature for biomimetic lubricants [73] that are recyclable and eco-friendly. As per the current research trend, biomimetic lubricants can be considered as the future of tribology. Recently, human synovial lubrication has been considered as one of the most plausible inspirations for biomimicry in lubrication technology [12]. The main reason for this is the remarkable life span of human lubrication. Furthermore, most of the 
investigations in the literature have indicated that the friction coefficient at the synovial joint is in the range $10.3-10.2$ [74] which makes it a viable candidate for automobile lubrication. On the other hand, surface modifications based on biological inspiration has been extensively noticed in literature. For instance, plants like Nepenthes pitcher plants and Salvinia have slippery surfaces that are being investigated for engineering applications [75]-[78]. These surfaces have enhanced lubrication capabilities along with the ability to withstand extreme conditions.

\section{Multiple features}

So far, it is evident that various nature-inspired techniques can be mimicked on automobiles by introducing different kinds of coatings. Moving a little bit further away, the recent research in literature has provided information in integrating multiple features with one coating. Liu et al. [79] have introduced one such coating that possesses multiple protective features such as self-healing, mechanical robustness, and mechanical gradients. Recently, there has been an interest to implement microstructures with multiple different biomimetic features at once [80]. The ultimate expectation of this research field is to develop a method to integrate self-cleaning, self-healing, drag reduction, and transparency into a single coating that can be applied to the automobile body. Currently, $\mathrm{TiO}_{2}$ can be considered as one of the most researched substances to implicate the multiple biomimetic features in automobiles [57]. This research includes functions such as transparency, self-cleaning, and photocatalytic degradation of the contaminants.

\section{DESIGN APPROACHES AND FUTURE DIRECTIONS}

Biomimicry based engineering design can be performed in two different approaches. They are: top-down approach and bottom-up approach. These approaches are depicted as a flow diagram in Figure 7.

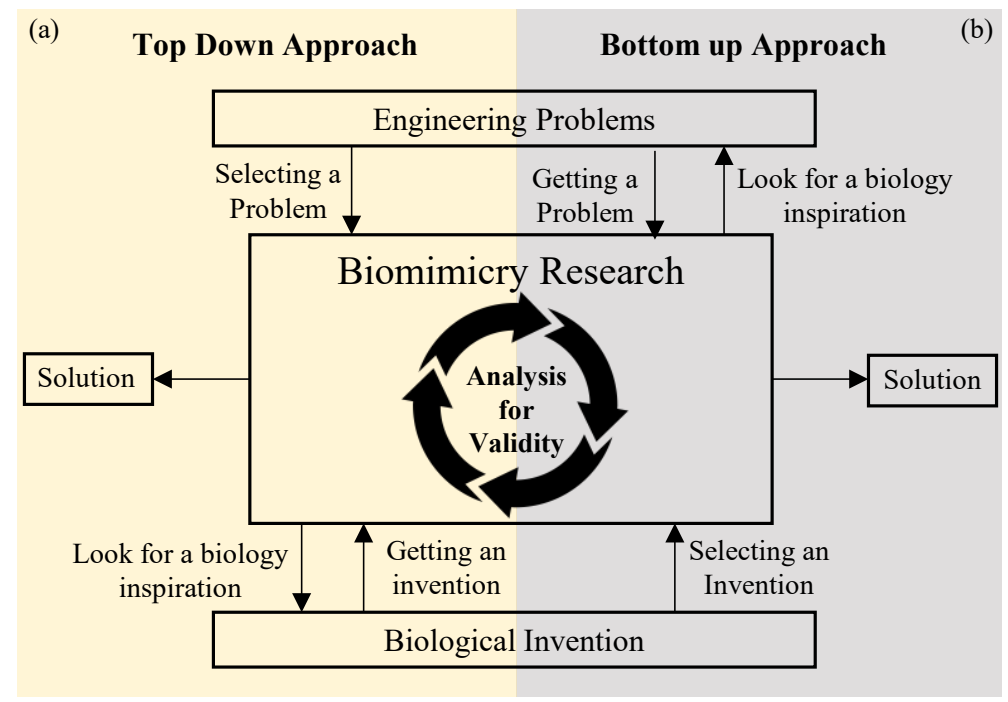

Figure 7. Major biomimetic design approaches in engineering: (a) top-down approach, (b) bottom-up approach.

In the first type, the top-down approach, a targeted engineering problem with a considerable research gap is selected and is addressed with different designs inspired by nature, see Figure 7(a). Then the nominated biomimetic designs will be analysed to verify their validity under altered conditions and compare them with state-of-the-art technology. If they were found to have innovative potential, then the process will proceed to develop a product with bionic properties. The bottom-up approach, on the other hand, starts from the structural findings in the bio-sciences, which are used to develop solutions for the existing research problems in engineering; see Figure 7(b). In this case, a variety of considerations such as material selection, fabrication method, design aspects, and cost are taken into account when evaluating the feasibility of using the discovered biological aspect in a particular engineering problem [81]. In both approaches, an iterative process is performed where different biological advancements can be linked to the different engineering research areas until the results suggest that the combination is ideal.

As per the analysis, it is obvious that the biomimetic designs have upgraded an abundance of automobile components by incorporating futuristic features. In a nutshell, biomimicry has given structural improvements to automobiles at both micro and macro levels. They cover vehicle body components, powertrain and under-the-hood components, and wheel assembly components. These improvements have reduced fuel consumption while increasing the performance of the automobile. Nevertheless, yet there are many sectors in automobile design that needs more attention but had been merely noticed in previous research. This includes the interior components, wheel assembly components, and under-hood components. Therefore, the authors witnessed a considerable research gap present in the selected scope. Thus, biomimetic solutions for automobile-related problems are yet to be discovered.

In automobile exterior designs, vehicles have extensively adopted numerous sophisticated shapes based on biomimicry. But in the design of under-the-hood mechanical components such as fans, ducts, heat exchangers are usually based on more conventional Cartesian geometries such as planes, cylinders, and spheres [5]. Therefore, these designs 
have large weights, and they take large packaging space in the engine bay. On the other hand, the vehicle interior components that interact with the passengers have merely been influenced by biomimicry. Although the exterior and powertrain of the vehicle mostly impact the performance of the vehicle, the interior of the vehicle provides the rider comfort and the easiness to handle the vehicle. As a result, the environment inside the vehicle also plays a major role in the purchase decision of an automobile. Hence, the interest in biomimetic related solutions for more sophisticated automobile interiors is expected in future developments.

In the sense of biological innovations, several findings can be used for automobiles that were not noticed in the automobile-related designs. One of them is the anti-wear mechanism that Mollusc shells (conchs) possess [82]. This is developed in seashells to act against the water-sand erosion on sandy beaches. This feature arises from a combination of their bio-tissue and their unique shape. The material of the Mollusc shell is a bio-ceramic composite, which has a complex microstructure resulting from biotic evolution for a thousand million years [55]. This is a potential structure for the offroad vehicle components.

Another incredible invention of biotechnology is mimicking the skin properties of the chameleon. It is a known fact that chameleon skin is capable of changing its colour. Therefore, extremely futuristic designs can be introduced by applying this technique to automobiles. Currently, literature provides evidence on research that modify microstructural properties of the magnesium alloys to obtain different colours in the surfaces while having rather robust mechanical properties [83]. However, there are more extensive approaches that can be initiated with this technology. For example, with this concept, automobile light intensity and colours can be changed based on environmental conditions. Even in the power generation, animals possess rather environmentally friendly and effective technology than any other alternative currently used in automobiles. This concept also has provisions to be investigated in future vehicles to support ecofriendly automobile development.

Based on the above facts, it is evident that areas of biology are still needed to be explored in the context of extracting biomimetic concepts on behalf of automobile engineering. Furthermore, the artificial intelligence-based systems that can optimised mechanical structures based on the given biological components should be investigated. As a result, more efficient and effective automobile-related components will be introduced.

\section{CONCLUSION}

This paper provides an extensive review of the-state-of-art biomimetic designs developed in the automobile area. The analysis of the paper was focused on the macro and micro scale mechanical structures that inspired the designs related to ground vehicles used in transportation. During the analysis, it was identified that even though there was heightened attention on innovations and inventions in biology, research related to these are usually published with more complex terminology that is not convenient for the research community working on automobile engineering. Because of this, the technology transfer between biology and engineering is monotonous. Hence, this article intends to become an inspiration for the automobile research community to create biomimetic design solutions.

This article provides classifications for the biomimetic research on automobiles in the defined scope. The classification highlights the biomimetic research on automobile engineering under two clusters: macroscale structures and microscale structures. So far, macroscale biomimetic structures have invaded automobile body, wheel assembly, powertrain and under-the-hood components. The microscale research was predominantly used to enhance the surface properties, lubrication and wear resistance. These developments are involved in increasing the performance of the vehicle, improving the fuel economy and incorporating additional features such as self-cleaning, self-healing, and wear resistance to future automobiles. When comparing the two clusters, in recent research, the microstructural implications have shown a higher involvement. The main reason for this is their ability of independent implementation. In other words, micro-structural products can be developed as coatings and include in automobile developments even after they are fully manufactured. Yet, the greatest effects of the biomimetics design approaches can be seen in the macro scale developments. Hence, the authors expect this article will become an inspiration for the researchers to be more involved in the macro-scale developments in the future.

This review on bio-inspired automobile design has led the authors to believe that natural life can provide incredible solutions, with a large diversity of designs, materials, and mechanical contrivances of every degree of complexity, for the engineering disciplines, including automobile development. Yet, during the review, the authors identified that the extensive involvement of automobiles in biomimetic designs had been unfortunately limited to the aerodynamic aspects. At present, the involvement of commercial vehicle manufacturers was also merely noticed in biomimetic creations. The main reason for this is the deficit of the research that can justify the effectiveness of the biomimetic concepts. However, in the future, with the use of biomimetics, there are provisions for developing rather optimised macro-structural components and micro-structural implications that provide multiple features in a single material. In conclusion, biomimicry can be considered as a promising technology, with an increasing trend, for future automobile developments. Therefore, the authors believe this review article will be extremely beneficial for the researchers in the automobile-related fields to understand new trends and harvest novel concepts for future research.

\section{REFERENCES}

[1] J. Hwang et al., "Biomimetics: Forecasting the future of science, engineering, and medicine," Int J Nanomedicine, vol. 10, no. 1, pp. 5701-5713, 2015, doi: 10.2147/IJN.S83642.

[2] J. K. Nagel et al., "Teaching bioinspired design using C-K theory," Bioinspired Biomim Nanobiomaterials, vol. 6, no. 2, pp. 
77-86, 2017, doi: 10.1680/jbibn.16.00013.

[3] E. Lurie-Luke, "Product and technology innovation: What can biomimicry inspire?," Biotechnol Adv, vol. 32, no. 8, pp. 14941505, 2014, doi: 10.1016/j.biotechadv.2014.10.002.

[4] G. M. Akash, "Application of biomimetics in design of vehicles - A review," Int Res J Eng Technol, vol. 7, no. 3, pp. 2753$2759,2020$.

[5] P. S. Fiske, J. Harman, and K. Shekar, "Lean, light and quiet: advances in automotive energy efficiency through biomimetic design," SAE Tech Pap, 2008-21-0028, 2008.

[6] Y. Gheraibia, K. Djafri, and H. Krimou, "Ant colony algorithm for automotive safety integrity level allocation," Appl Intell, vol. 48, no. 3, pp. 555-569, 2017, doi: 10.1007/s10489-017-1000-6.

[7] N. Chowdhary and P. D. Kaur, "Dynamic route optimisation using nature-inspired algorithms in IoV," in Proceedings of First International Conference on Smart System, Innovations and Computing, 2018, pp. 495-504, doi: 10.1007/978-981-10-5828847.

[8] J. Y. Potvin, A review of bio-inspired algorithms for vehicle routing, vol. 161. Berlin, Heidelberg: Springer, 2009.

[9] M. A. Mellal and E. J. Williams, "Parameter optimisation of advanced machining processes using cuckoo optimisation algorithm and hoopoe heuristic," J Intell Manuf, vol. 27, no. 5, pp. 927-942, 2016, doi: 10.1007/s10845-014-0925-4.

[10] C. P. Igiri, D. Bhargava, and Y. Singh, "A penalty method based-spring design optimisation using bio-inspired computation approach," in Proceedings - 2019 Amity International Conference on Artificial Intelligence, AICAI 2019, 2019, pp. 182-187, doi: 10.1109/AICAI.2019.8701248.

[11] S. M. Mota et al., "Bio-inspired motion detection for a blind spot overtaking monitor," Int J Robot Autom, vol. 19, no. 4, pp. 190-196, 2004, doi: 10.2316/journal.206.2004.4.206-2713.

[12] A. Siddaiah and P. L. Menezes, “Advances in bio-inspired tribology for engineering applications,” J Bio- Tribo-Corrosion, vol. 2, no. 4, 2016, doi: 10.1007/s40735-016-0053-0.

[13] A. Du Plessis et al., "Beautiful and functional: A review of biomimetic design in additive manufacturing," Addit Manuf, vol. 27, pp. 408-427, 2019, doi: 10.1016/j.addma.2019.03.033.

[14] C. Riley, "17 iconic cars matched with photos of the animals they are named after," Autowise, 2016 [Online], Available: https://autowise.com/17-iconic-cars-matched-with-photos-of-the-animals-they-are-named-after/.[Accessed: Jan 15, 2021].

[15] M. Hou and L. Fu, "On auto bionic modeling design study based on cognitive psychology theories," in Proceedings 2011 International Conference on Mechatronic Science, Electric Engineering and Computer, MEC 2011, 2011, pp. 1641-1644, doi: 10.1109/MEC.2011.6025793.

[16] H. Chowdhury et al., "Design of an energy efficient car by biomimicry of a boxfish," in 2nd International Conference on Energy and Power, 2018, pp. 40-44, doi: 10.1016/j.egypro.2019.02.116.

[17] S. Peng et al., "Application of bionics of tiger beetle to aerodynamic optimisation of MIRA fastback model," in International Conference on Computer Science and Application Engineering, 2017, pp. 645-654, doi: 10.12783/dtcse/csae2017/17537.

[18] I. K. Agyei et al., "Avocado pear as a source of inspiration for the design of a model truck as a desk organiser," Arts Des Stud, vol. 86, pp. 40-52, 2020, doi: 10.7176/ADS/86-05.

[19] C. Diels, A. Siamatas, and C. Johnson, "Designing for the new vehicle DNA: Evaluation of the MAYA principle for the rapid adoption of sustainable vehicles," 2013, doi: 10.13140/RG.2.1.2882.2889.

[20] "The dimpled future of cars," Automology, 2014. [Online], Available: https://www.automology.com/the-dimpled-future-ofcars/. [Accessed: Jan 15, 2021].

[21] J. Neff, "Lexus debuts dimpled LS 460 L for US Open," $2009 . \quad$ [Online], Available: https://www.autoblog.com/2009/06/18/lexus-orders-dimpled-ls460-l-for-u-s-open/. [Accessed: Jan 15, 2021].

[22] P. C. Salaverry and T. Dallas, "Vehicle with drag-reducing outer surface," US 7,810,867 B2, 2010.

[23] "Extreme aerodynamics due to unique roof surface," Futuristic News. .

[24] V. Kashyap et al., "Aerodynamic effect of aspect ratio of spherical depressions on the bonnet of hatchback cars," SAE Tech Pap, 2019, doi: 10.4271/2019-01-5096.

[25] L. Shyamal, "The hidden world of the crouching tiger-beetles," Catching Flies, 2020. .

[26] J. J. Kim, J. Hong, and S. J. Lee, "Bio-inspired cab-roof fairing of heavy vehicles for enhancing drag reduction and driving stability," Int J Mech Sci, vol. 131-132, pp. 868-879, 2017, doi: 10.1016/j.ijmecsci.2017.08.010.

[27] H. Ghaffar et al., "A simulation study of tubercles effect of aerodynamics performance on car rear spoiler," J Phys Conf Ser, 2019, doi: 10.1088/1742-6596/1349/1/012034.

[28] F. E. Fish, "Biomimetics: determining engineering opportunities from nature," In Proc. SPIE 7401, Biomimetics and Bioinspiration, 2009, pp. 740109, doi: 10.1117/12.824106.

[29] C. Guo et al., "Structural design inspired by beetle elytra and its mechanical properties," Chin Sci Bull, vol 57, pp. 941-947, 2012, doi: 10.1007/s11434-011-4956-3.

[30] D. Kim et al., "A bio-inspired device for drag reduction on a three-dimensional model vehicle," Bioinspiration and Biomimetics, vol. 11, no. 2, 2016, doi: 10.1088/1748-3190/11/2/026004.

[31] X. M. Fan et al., "Study on automobile body performance of honeycomb sandwich composite material," Adv Mater Res, vol. 567, pp. 146-149, 2012, doi: 10.4028/www.scientific.net/AMR.567.146.

[32] Z. Wang et al., "On the crashworthiness of bio-inspired hexagonal prismatic tubes under axial compression," Int J Mech Sci, vol. 186, p. 105893, 2020, doi: 10.1016/j.ijmecsci.2020.105893.

[33] C. Hundertmark et al., "Diatom-inspired Plastic Deformation Elements for Energy Absorption in Automobiles," J Bionic Eng, vol. 12, no. 4, pp. 613-623, 2015, doi: 10.1016/S1672-6529(14)60151-7.

[34] O. A. Ganilova and J. J. Low, "Application of smart honeycomb structures for automotive passive safety," Proc Inst Mech Eng Part D J Automob Eng, vol. 232, no. 6, pp. 797-811, 2018, doi: 10.1177/0954407017708916.

[35] T. Wu et al., "Research on non-pneumatic tire with gradient anti-tetrachiral structures," Mech Adv Mater Struct, 2020, doi: $10.1080 / 15376494.2020 .1734888$.

[36] A. Mohan et al., "Design and analysis of non-pneumatic tyre," IOP Conf Ser Mater Sci Eng, vol. 263, no. 6, 2017, doi: 10.1088/1757-899X/263/6/062061.

[37] M. M. Pewekar and S. D. Gaikwad, "Strength validation of hexagonal cellular spoked non-pneumatic tires for automobiles through finite element analysis," Int J Sci Res Sci Technol, vol. 4, no. 5, pp. 1044-1055, 2018. 
[38] L. Ivanović et al., "Biomimetics design for tribological applications," Tribol Ind, vol. 40, no. 3, pp. 448-456, 2018, doi: 10.24874/ti.2018.40.03.11.

[39] W. J. P. Barnes et al., "Bionics and wet grip," Tire Technol Int, no. October, pp. 56-60, 2002.

[40] S. Kaluvan, C. Y. Park, and S. B. Choi, "Bio-inspired device: A novel smart MR spring featuring tendril structure," Smart Mater Struct, vol. 25, no. 1, 2016, doi: 10.1088/0964-1726/25/1/01LT01.

[41] H. T. Yang et al., "Preliminary design, experiment, and numerical study of a prototype hydraulic bio-inspired damper," $J$ Sound Vib, vol. 459, 2019, doi: 10.1016/j.jsv.2019.07.011.

[42] G. E. Fantner et al., "Sacrificial bonds and hidden length dissipate energy as mineralized fibrils separate during bone fracture," Nat Mater, vol. 4, no. 8, pp. 612-616, 2005, doi: 10.1038/nmat1428.

[43] Dave Jenike, "Cheetah Running," Flickr, 2010. .

[44] L. Chen et al., "Bionic innovation design of disc brake," Int J Interact Des Manuf, vol. 14, no. 1, pp. 309-322, 2020, doi: 10.1007/s12008-020-00657-w.

[45] Z. Wang et al., "Novel bionic design method for skeleton structures based on load path analysis," Appl Sci, vol. 10, no. 22, 2020, doi: 10.3390/app10228251.

[46] E. Kalm, "Design of an aerodynamic green car," M.S. thesis, Lulea University of Technology, Sweeden, 2007.

[47] S. Wang et al., "Noise reduction of automobile cooling fan based on bio-inspired design," Proc Inst Mech Eng Part D J Automob Eng, 2020, doi: 10.1177/0954407020959892.

[48] Y. Nakanishi et al., "Bio-inspired seal lip for application in electric vehicle coolant pumps," Mech Eng Lett, vol. 4, 2018, doi: 10.1299/mel.17-00654.

[49] S. Ki et al., "A bio-inspired, low pressure drop liquid cooling system for high-power IGBT modules for EV/HEV applications," Int J Therm Sci, p. 106708, 2020, doi: 10.1016/j.ijthermalsci.2020.106708.

[50] B. Shi, A. Elsayed, and S. Mahmoud, "CFD simulation of honeycomb adsorption bed for automotive cooling system," 2014.

[51] J. Galindo et al., "Heat transfer modelling in honeycomb wall-flow diesel particulate filters," Energy, vol. 43, no. 1, pp. 201213, 2012, doi: 10.1016/j.energy.2012.04.044.

[52] S. P. Dalawai et al., "Recent advances in durability of superhydrophobic self-cleaning technology: A critical review," Prog Org Coatings, vol. 138, no. May 2019, 2020, doi: 10.1016/j.porgcoat.2019.105381.

[53] K. B. Golovin et al., "Bioinspired surfaces for turbulent drag reduction," Philos Trans R Soc A Math Phys Eng Sci, vol. 374 , 2016, doi: 10.1098/rsta.2016.0189.

[54] C. Nanotech, "Motorsport." 2020 [Online], Available: https://www.choosenano.com/solutions/motorsport/. [Accessed: July 19, 2021].

[55] R. A. Singh and E.-S. Yoon, "Biomimetics in tribology - Recent developments," J Korean Phys Soc, vol. 52, no. 3, pp. 656668, 2008, doi: 10.3938/jkps.52.656.

[56] S. Nishimoto and B. Bhushan, "Bioinspired self-cleaning surfaces with superhydrophobicity, superoleophobicity, and superhydrophilicity," RSC Adv, vol. 3, no. 3, pp. 671-690, 2013, doi: 10.1039/c2ra21260a.

[57] N. T. Padmanabhan and H. John, "Titanium dioxide based self-cleaning smart surfaces: A short review," J Environ Chem Eng, vol. 8, no. 5, 2020, doi: 10.1016/j.jece.2020.104211.

[58] Nissan, "Self Cleaning Paint Concept." 2014 [Online], Available: https://www.nissanusa.com/experience-nissan/news-andevents/self-cleaning-car-paint.html. [Accessed: Jan 15, 2021].

[59] Sym-Tech, "Next Generation Interior and Exterior Protection." 2018 [Online], Available https://www.symtech.ca/en/services/sharkskinr [Accessed: Jan 15, 2021].

[60] S. Thakur, "Sustainable nanostructural materials for shape memory, self-healing, and self-cleaning applications," in Dynamics of Advanced Sustainable Nanomaterials and Their Related Nanocomposites at the Bio-Nano Interface, Elsevier Inc., 2019, pp. 235-250.

[61] Y. Liu et al., "Biomimetic hydrophobic surface fabricated by chemical etching method from hierarchically structured magnesium alloy substrate," Appl Surf Sci, vol. 280, pp. 845-849, 2013, doi: 10.1016/j.apsusc.2013.05.072.

[62] T. Xiang et al., "Slippery liquid-infused porous surface for corrosion protection with self-healing property," Chem Eng $J$, vol. 345, pp. 147-155, 2018, doi: 10.1016/j.cej.2018.03.137.

[63] C. Seubert et al., "An overview of the scratch resistance of automotive coatings: Exterior clearcoats and polycarbonate hardcoats," Coatings, vol. 2, no. 4, pp. 221-234, 2012, doi: 10.3390/coatings2040221.

[64] R. Taurino et al., "Preparation of scratch resistant superhydrophobic hybrid coatings by sol-gel process," Prog Org Coatings, vol. 77, no. 11, pp. 1635-1641, 2014, doi: 10.1016/j.porgcoat.2014.05.009.

[65] M. Groenewolt, "Highly scratch resistant coatings for automotive applications," Prog Org Coatings, vol. 61, no. 2-4, pp. 106-109, 2008, doi: 10.1016/j.porgcoat.2007.07.036.

[66] S. K. Ghosh, Self healing materials - Fundamentals design strategies and applications. Wiley-Vch Verlag GmbH \& Co. KGaA, 2009.

[67] "Lamborghini and MIT unveil self-healing concept car," The Engineer, 2017. [Online], Available: https://www.theengineer.co.uk/lamborghini-mit-self-healing-concept-car/. [Accessed: Jan 15, 2021] .

[68] "Scratch shield," Nissan Motor Corporation. 2020 [Online], Available: https://www.nissanglobal.com/EN/TECHNOLOGY/OVERVIEW/scratch.html. [Accessed: Jan 15, 2021].

[69] H. Zhou et al., "The thermal fatigue resistance of cast iron with biomimetic non-smooth surface processed by laser with different parameters," Mater Sci Eng A, vol. 428, pp. 141-147, 2006, doi: 10.1016/j.msea.2006.04.101.

[70] H. Yang et al., "The relationship between the model of the laser biomimetic strengthening of gray cast iron and matching between different brake pads," Metals (Basel), vol. 10, no. 2, 2020, doi: 10.3390/met10020184.

[71] L. Ren and Y. Liang, "Biological couplings: Classification and characteristic rules," Sci China, Ser E Technol Sci, vol. 52, no. 10, pp. 2791-2800, 2009, doi: 10.1007/s11431-009-0325-8.

[72] S. Kamat et al., "Structural basis for the fracture toughness of the shell of the conch strombus gigas," Nature, vol. 405, pp. 1036-1040, 2000, doi: 10.1038/35016535.

[73] A. Morina, T. Liskiewicz, and A. Neville, "Designing new lubricant additives using biomimetics," WIT Trans Ecol Environ, vol. 87, pp. 157-166, 2006, doi: 10.2495/DN060151.

[74] A. Neville et al., "Synovial joint lubrication - Does nature teach more effective engineering lubrication strategies?," Proc Inst 
Mech Eng Part C J Mech Eng Sci, vol. 221, no. 10, pp. 1223-1230, 2007, doi: 10.1243/09544062JMES724.

[75] X. Li et al., "Salvinia-like slippery surface with stable and mobile water/air contact line," Natl Sci Rev, 2020, doi: 10.1093/nsr/nwaa153.

[76] T. S. Wong et al., "Bioinspired self-repairing slippery surfaces with pressure-stable omniphobicity," Nature, vol. 477, pp. 443-447, 2011, doi: 10.1038/nature10447.

[77] Z. Dong et al., "Superoleophobic slippery lubricant-infused surfaces: combining two extremes in the same surface," $A d v$ Mater, vol. 30, no. 45, 2018, doi: 10.1002/adma.201803890.

[78] J. Yong et al., "Nepenthes inspired design of self-repairing omniphobic slippery Liquid infused porous surface (SLIPS) by femtosecond laser direct writing," Adv Mater Interfaces, vol. 4, no. 20, 2017, doi: 10.1002/admi.201700552.

[79] Z. Liu et al., "Biomimetic materials with multiple protective functionalities," Adv Funct Mater, vol. 29, no. 28, 2019, doi: 10.1002/adfm.201901058.

[80] P. P. Vijayan and D. Puglia, "Biomimetic multifunctional materials: A review," Emergent Mater, vol. 2, no. 4, pp. 391-415, 2019, doi: 10.1007/s42247-019-00051-7.

[81] R. Bannasch, "Bionics," in Technology Guide Principles-Applications-Trends, Springer, 2013, pp. $178-183$.

[82] L. Tian, X. Tian, G. Hu, Y. Wang, and L. Ren, "Effects and mechanisms of surface topography on the anti-wear properties of molluscan shells (Scapharca subcrenata) using the fluid-solid interaction method," Sci World J, vol. 2014, pp. 185370. , 2014, doi: $10.1155 / 2014 / 185370$

[83] L. Liu et al., "Constructing colorful surfaces with mechanical robustness for magnesium alloys via a reagent-free method," ACS Appl Mater Interfaces, vol. 12, pp. 48206-48215, 2020, doi: 10.1021/acsami.0c14359.

[84] A. Baier and J. Nojeck, "Possible application of artificial shark skin as surface reducting aerodynamic drag," Sel Eng Probl, vol 7, pp. 5-8, 2016. 